\title{
Do Positive Psychology Interventions Impact on the Subjective Wellbeing and Depression of Clients? A Systematic Methodological Review
}

\author{
Kara McTiernan ${ }^{1,2} \cdot$ Fiona Gullon-Scott ${ }^{2} \cdot$ Robert Dudley $^{2,3}$
}

Accepted: 7 September 2021 / Published online: 26 November 2021

(c) The Author(s) 2021

\begin{abstract}
Mental health services are placing a greater emphasis on wellbeing and recovery. The current research investigated if positive psychology interventions (PPIs) increase peoples' subjective wellbeing and reduce clinical depression. A systematic methodological review was conducted on randomized-control-trials with people attending clinical services. Five databases were searched. A hand search was then completed on the reference lists of the identified articles and the associated journals. Eleven research interventions were reviewed. PPIs were found to significantly increase wellbeing, relative to controls and there were fewer studies indicating a difference in decreasing depression. However, subsequent analysis revealed that the interventions were heterogeneous which limits the drawing of definitive systematic conclusions. A methodological evaluation also found that there were recurring issues: in delivering the interventions, measuring subjective wellbeing, and applying the design. Thus, the methodological quality of the research interventions, as measured by the current review was low. There is emerging evidence that PPIs improve peoples' mental health. However, there is scope to standardize and to improve the quality of the research interventions.
\end{abstract}

Keywords Positive psychology $\cdot$ Subjective wellbeing $\cdot$ Clinical depression $\cdot$ Randomised control trials $\cdot$ Systematic methodological review

\section{Introduction}

\section{Positive Psychology}

Positive psychology is the scientific study of positive: subjective experiences, individual traits and, institutions (Seligman \& Csikszentmihalyi, 2000). It provides a theoretical basis to improve peoples' wellbeing (Seligman, 2002). It is recognised that mental health difficulties and mental health

Kara McTiernan

karamctiernan@gmail.com

1 North Dublin Mental Health Services, Health Service Executive, Swords Health Centre, Bridge Street, Swords, Co. Dublin, Ireland

2 School of Psychology, Newcastle University, 4th Floor, Dame Margaret Barbour Building, Wallace Street, Newcastle Upon Tyne NE2 4DR, UK

3 Gateshead Early Intervention in Psychosis Service, CNTW NHS Foundation Trust, Dryden Centre, Evistones Road, Newcastle upon Tyne NE9 5UR, UK are related but separate entities (Payton, 2009). Positive psychology therefore stipulates that even in times of difficulty people can work towards and attain a sense of wellbeing (Compton \& Hoffman, 2012). Cultivating this sense of wellbeing can in turn psychologically strengthen people which can assist with the management of difficulties. Moreover, Peterson (2006) highlights that mental health is more than just the alleviation of symptom distress, it also encompasses the concept of living well.

Positive psychology constructs align with the work of Rogers (1961) and Maslow (1943) with their postulations of unconditional positive regard and self-actualization. Frankl's (1946) demonstration of enduring pain, creating meaning, and developing insight also exemplifies growing psychologically from trauma. A distinguishable feature of the current positive psychology paradigm is that it is derived from the discipline of clinical psychology. There is therefore the commitment to the development of an empirical evidencebase and to actively changing maladaptive cognitions and behaviours into adaptive ones. However, in contrast with the majority of psychotherapies, which centre on dysfunction 
and the alleviation of symptom distress, positive psychotherapy attends: to the positives within people, to the building of psychological resources and to increasing subjective wellbeing (Seligman \& Rashid, 2014). The topic of subjective wellbeing in itself sparked philosophers' conceptualizations of eudaimonia and hedonism, and there is some investigation of the construct within psychology (Fordyce, 1977). Positive psychotherapy is also defined by attending positively to the persons' personality or sense of self by activating character strengths or positive traits (Peterson \& Seligman, 2004). Thus, in line with the ethos of recovery, the person is facilitated to work on their authentic sense of self, to generate subjective wellbeing, which in turn potentially enables the re-emergence of a well-being or a person who is well.

\section{Positive Psychology, Subjective Wellbeing and Depression}

The seminal positive psychology intervention by Seligman et al. (2005) was underpinned by the authentic happiness theory (Seligman, 2002) which proffers that subjective wellbeing can be attained through three processes: positive emotion (the pleasant life), engagement (the engaged life) and meaning (the meaningful life). Seligman (2012) has since broadened the theory to include positive relationships and accomplishment, as encapsulated by the PERMA model. A consensus has emerged that subjective wellbeing consists of a cognitive evaluation of life satisfaction in conjunction with high positive affect and low negative affect (Diener et al., 2017). Despite this, numerous measures of subjective wellbeing remain within the literature (Cooke et al., 2016). Seligman et al. (2005) investigated the use of five positive psychology interventions (PPIs) in generating subjective wellbeing, namely: (1) identifying your signature strengths (2) using your signature strengths in a new way (3) identifying three good things in life (4) reflecting on you at your best (5) and expressing gratitude, online with the general public $(\mathrm{N}=411)$. The 'using strengths', 'three good things', and 'gratitude' PPIs led to significantly higher levels of happiness, as measured by the Authentic Happiness Inventory (AHI) (Peterson, 2005) and significantly lower levels of depression, as measured by the Center for Epidemiological Studies Depression Scale (CESD) (Radloff, 1977) relative to the control. However, the PPIs of 'identifying strengths' and 'reflecting on you at your best' did not result in significant changes in happiness or depression over time. Gander et al. (2013) replicated the majority of the 'using your strengths', 'three good things' and 'gratitude' findings $(\mathrm{N}=622)$. However, the 'three good things' PPI did not differ from the control in reducing depression. Similarly, Mongrain and Anselmo-Matthews (2012) replicated the 'using your strengths' and 'three good things' happiness findings $(\mathrm{N}=344)$, but the PPIs did not differ from the control in alleviating depression.

Two meta-analyses (Bolier et al., 2013; Sin \& Lyubomirsky, 2009) concluded that PPIs increase wellbeing and decrease depression with small to medium effect sizes. However, it was noted that the studies varied in quality and incorporated different theories and interventions, including Ryff's wellbeing theory (Fava et al, 2005) and coaching (Green et al, 2006). Santos et al. (2013) also reviewed a variety of PPIs and found that the interventions: improved wellbeing, reduced depression and prevented relapse. However, there remains a lack of systematic evidence for applying the Seligman et al. (2005) PPIs in clinical practice (Johnson \& Wood, 2017). Clinically, the Seligman et al. (2005) PPIs were initially delivered with people with depression.

\section{Clinical Depression and Positive Psychotherapy}

Depression is defined by the presence of negative affect and in accordance with Beck's model (1979) it is characterized by a triad of negative cognitions about: oneself, the world and the future. Positive psychotherapy may therefore be an effective intervention for depression considering that: positive emotion, engagement and meaning alongside the associated generation of positive cognitions and affect are the opposite of the defining features of depression (Seligman et al., 2006). Activating people through the generation of positive cognition also logically builds on learned helplessness theory (Seligman, 1972). This stipulates that when people are repeatedly exposed to adversity many begin to experience negative cognitions and to believe that personal control cannot be exerted on the environment and so become deactivated. It is noteworthy that depression is comorbid with many mental health difficulties and this may be indicative of a unifying cognitive or affective process present throughout mental health difficulties (Querstret \& Cropley, 2013), such as negative cognition or negative affect. Positive psychotherapy may therefore be beneficial to people with a range of clinical presentations and it has a role more broadly in generally facilitating peoples' subjective wellbeing. It is noted that depression significantly reduces life satisfaction (Vazquez et al., 2015) and that the subjective wellbeing of people with depression is lower than that of people not attending clinical services (Watson \& O'Hara, 2017). Currently, there is conflicting evidence with regards to whether depression and subjective wellbeing are at polar ends of the same continuum (Wood et al., 2010) or if negative affect and positive affect are distinctive constructs (Bradburn, 1969). Nevertheless, the evidence indicates that positive affect facilitates emotional regulation (Tugade \& Fredrickson, 2004). 


\section{The Current Review}

Previous reviews revealed issues in the quality of PPI studies and incorporated various theories, different types of wellbeing as well as both general and clinical populations. A systematic methodological review therefore specifically investigated the Seligman et al. (2005) PPIs as applied in clinical practice to subjective wellbeing and clinical depression.

\section{Methods}

The current review systematically evaluated the evidence to answer the question of: do PPIs increase the subjective wellbeing and reduce the clinical depression of people attending clinical services? The PRISMA guidelines (Moher et al., 2009) informed the development of the protocol for the manual and database searches.

\section{Eligibility Criteria}

\section{Population}

The population was limited to adults with clinical depression attending: mental health, physical health, learning disability, forensic and older adult services. No restrictions were placed on: whether depression was the primary or secondary presentation, the number of episodes of depression or the person's length of time attending the service.

\section{Intervention}

It was a requirement for interventions to have included at least one of the: 'identifying strengths', 'using strengths' 'three good things', 'you at your best' and/or 'gratitude' PPIs (Seligman et al., 2005). Interventions could be delivered individually or in group, with at least one session in person. The latter reflects the usual practice of people with depression developing an in person therapeutic relationship.

\section{Type of Study}

The research was limited to randomized control trials (RCT), as this is the gold standard of treatment outcome research (Field \& Cartwright-Hatton, 2015). It is also the most frequently applied method to test the efficacy of novel clinical interventions (Nathan \& Gorman, 2007), and this is supported by NICE (Guy et al., 2012). No limits were placed on the control group which could be: no treatment, treatment as usual (TAU), placebo or other treatment.

\section{Outcome Measures}

It was a requirement for studies to include self-report measures of subjective wellbeing and depression. In line with the agreed definition of subjective wellbeing, the search was receptive to the Satisfaction With Life Scale SWLS (Diener et al., 1985) and to the Positive and Negative Affect Schedule PANAS (Watson et al., 1988) in addition to measures labelled wellbeing and/or happiness. Clinical depression must have been recorded on the measure of depression.

\section{Date range and Publication Status}

Research interventions undertaken from 2005 onwards, published in peer reviewed journals were reviewed.

\section{Search Terms}

Online pilot searches were conducted on the broad term 'positive psychology interventions'. This phrase was searched in order to elicit the key terms associated with the interventions such as 'strengths' and 'subjective wellbeing'. Systematic reviews (Cuijpers et al., 2013; Liebherz et al., 2017) were utilized to identify the key terms associated with depression. The final search terms were Positive psychology OR Positive psychology intervention* OR positive psychotherapy AND depress* OR dysthymi* OR low mood. Wellbeing was not a search term as it limited the scope of the papers.

\section{Databases, Search Strategy and Screening}

A systematic literature search was conducted on five databases: PsychINFO (accessed via Ovid: SP), MEDLINE (accessed via Ovid: SP), EMBASE (assessed via Ovid: SP) and Scopus (accessed via Elsevier's SciVerse platform). An additional search was undertaken within the Cochrane Library. The key terms were adapted for each database and were searched for in the title, abstract and keywords of the text. The final search was conducted on the 6th of January 2018. This was repeated on the HSE e-library on the 22nd of December 2020.

Nine hundred and thirty-eight articles emerged from the initial search strategy, which were exported to endnote (version seventeen). The articles were screened for duplication. Following this, the titles, and abstracts of the remaining five hundred and seventy articles were screened to ensure that they related to the research question. The full texts of the remaining forty-one articles were then reviewed to confirm adherence to the eligibility criteria. Nine articles remained 
following this screening process. The repeat search resulted in two additional papers.

\section{Hand Search}

The reference lists of the final articles were checked. The associated journals were also searched. This did not result in the emergence of any additional interventions.

\section{Additional Search Strategies}

Positive psychology websites including: the authentic happiness website (www.authentichappiness.sas.upenn.edu), the VIA strengths website (www.viacharacter.org), the positive psychology research centre (ppc.sas.upenn.edu/ research/positive-psychology-research) and the UK positive psychology forum (www.positivepsychology.org.uk) were searched. Google scholar also identified the papers citing the seminal positive psychology study. This included two metaanalyses of positive psychology interventions (Bolier et al., 2013; Sin \& Lyubomirsky, 2009) three systematic reviews in mental health services (Santos et al., 2013; Schrank et al., 2013; Walsh et al., 2017) four systematic reviews in physical health services (Casellas-Grau et al., 2014; Iddon et al., 2016; Macaskill, 2016; Ghosh \& Deb, 2017) and two practice reviews (Hone et al., 2015; Rashid, 2015). These reviews were checked, alongside a review by Chakhssi et al (2018). Google scholar also retrieved the papers citing the initial trial of positive psychotherapy for clinical depression (Seligman et al, 2006) and the website clinicaltrials.gov was scanned. Additionally, a hand search was completed on the special issues of positive psychology published within the Psychologist (Linley et al., 2003), the Clinical Psychology Review (Wood \& Tarrier, 2010) and the Counselling Psychologist (Magyar-Moe et al, 2015).

One of the authors was aware of a research intervention which drew on PPIs, which was not detected by the search strategy. This intervention was designed for clients with persecutory delusions as opposed to depression. However, as it met the eligibility criteria it was included in the review. The final findings are illustrated in Fig. 1.

\section{Results}

Table 1 details that nine studies reported differences in increasing wellbeing and five studies found differences in reducing depression, with medium to large effect sizes. The degree of change in depression ranged from moderate to normal levels (Seligman et al., 2006), severe to moderate levels (Freeman et al., 2014), moderate to mild levels (Dowlatabadi et al., 2016) moderate to normal levels (Taylor et al., 2016) and from moderate to normal levels (Furchtlehner et al., 2019). However, the interventions differed on: the primary presentation, the levels of depression, the controls, the content, the format, the intensity of the intervention and the measures utilized. This limits the value of any overall estimate of effect size and it could also account for the emergence of inconsistent results. A methodological evaluation was subsequently undertaken in order to evaluate the quality of the studies.

\section{Development of an Evaluation Grid}

An evaluation grid was developed by amalgamating general criteria for evaluating research with criteria that is specific to RCTs and research interventions. This involved integrating the Kazdin guidelines (1995), the CONSORT guidelines (Schulz et al., 2010) and the Jadad measure (Jadad et al, 1996). The latter is the most reliable and valid measure for assessing RCTs (Olive et al., 2008). The Gearing Guidelines (2011) and the Medical Research Council Guidelines (2000) were also utilized to ensure sensitivity to evaluating the interventions. The final items reflected the research question and were reviewed and modified by each of the authors. The resulting grid is available on request from the first author. Each item was broadly scored on a scale ranging from 0 (not addressed), 1 (partially addressed with major issues that compromise the research), 2 (partially addressed with minor issues that compromise the research) to 3 (fully addressed). Items relating to the theory, interventions and measures were double weighted, as these are central components in appraising therapeutic interventions (Rychetnik et al., 2002). The quality scores of the studies could range from $0(0 \%)$ to $240(100 \%)$, with a higher score indicating greater quality.

\section{An Evaluation of the Studies}

One of the authors applied the evaluation grid to the interventions. The average quality score of the interventions was 95 (39\%). The methodological quality of the research interventions, as evaluated by the current review, was therefore low. The results from the higher quality studies did not provide a clear pattern in relation to outcomes. However, a higher quality study (Furchtlehner et al., 2019) found a difference between PPI and CBT in increasing wellbeing and decreasing depression, with PPI resulting in greater gains. Another higher quality research intervention (Chaves et al., 2016) found no difference between CBT and PPI in increasing wellbeing and decreasing depression, with both resulting in improvements. In considering the research interventions collectively there were recurring methodological issues in: detailing the theoretical underpinning of the studies, 


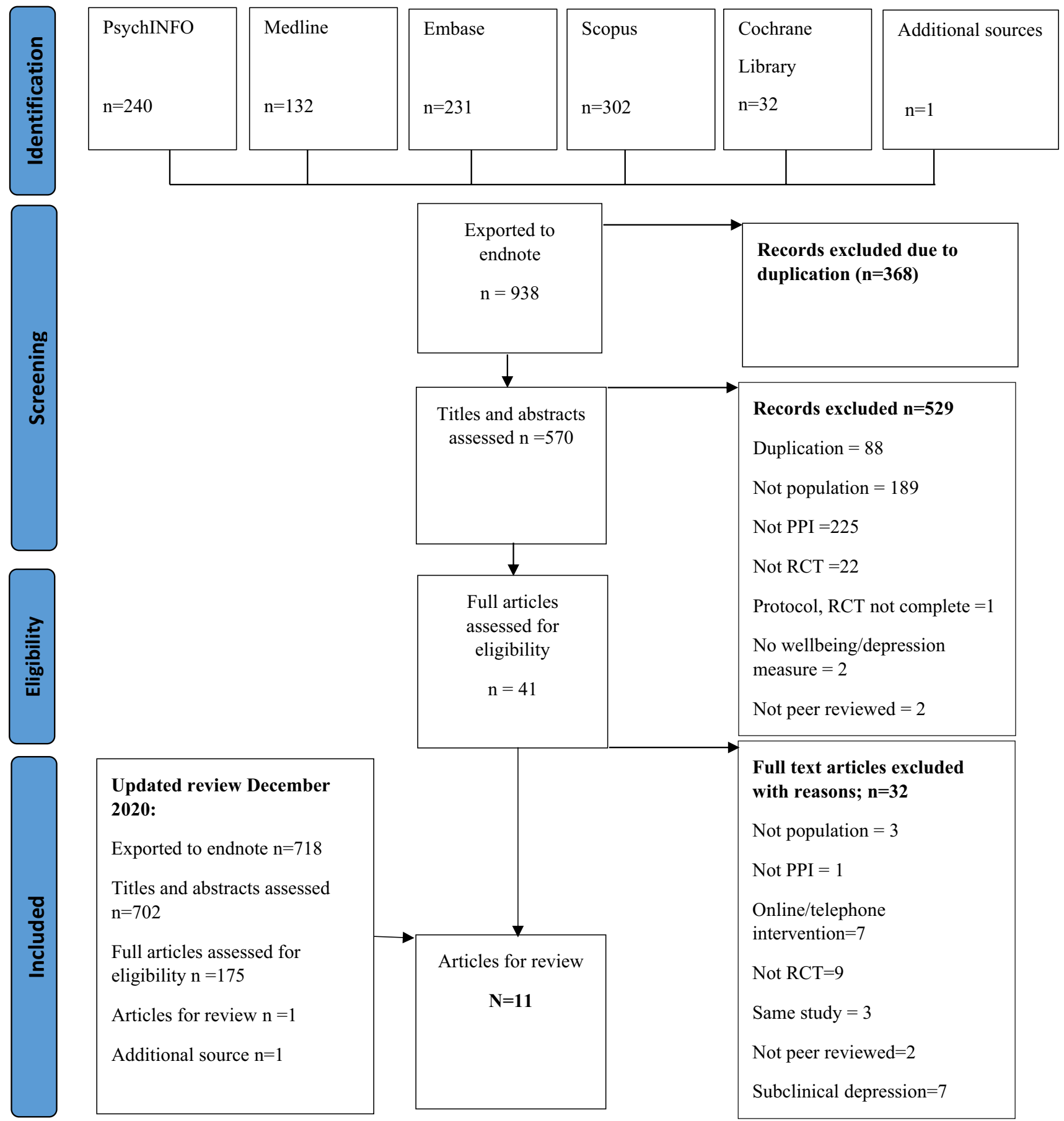

Fig. 1 The PRISMA flowchart illustrating the screening process

delivering the interventions, measuring subjective wellbeing and in implementing the RCT design.

In considering the theoretical underpinning of the research interventions there was a lack of a conceptual linking between the constructs. Seligman et al. (2006) and Furchtlehner et al. (2019) delineated the authentic happiness theory/ PERMA model alongside its evidence-base. Three studies alluded to elements of positive psychology theory, with the remaining research interventions not presenting a clear theory. A comprehensive definition of wellbeing was also absent. Seligman et al. (2006) and Pietrowsky and Mikutta (2012) provided definitions that were not explicitly linked to the intervention or to all of the measures. For instance, Pietroswsky and Mikutta (2012) operationalized wellbeing as consisting of high positive affect, low negative affect and life satisfaction. Two research interventions 


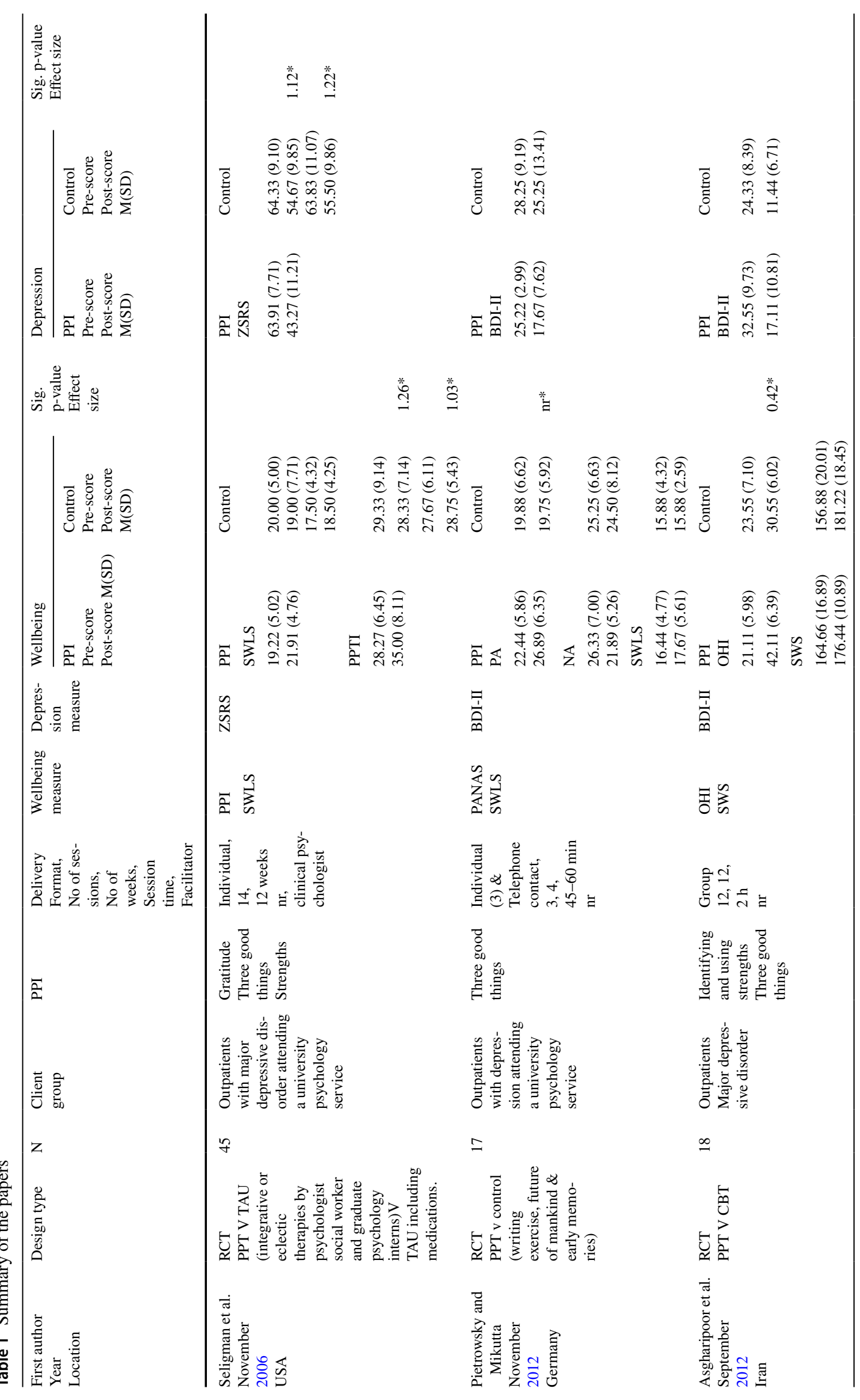




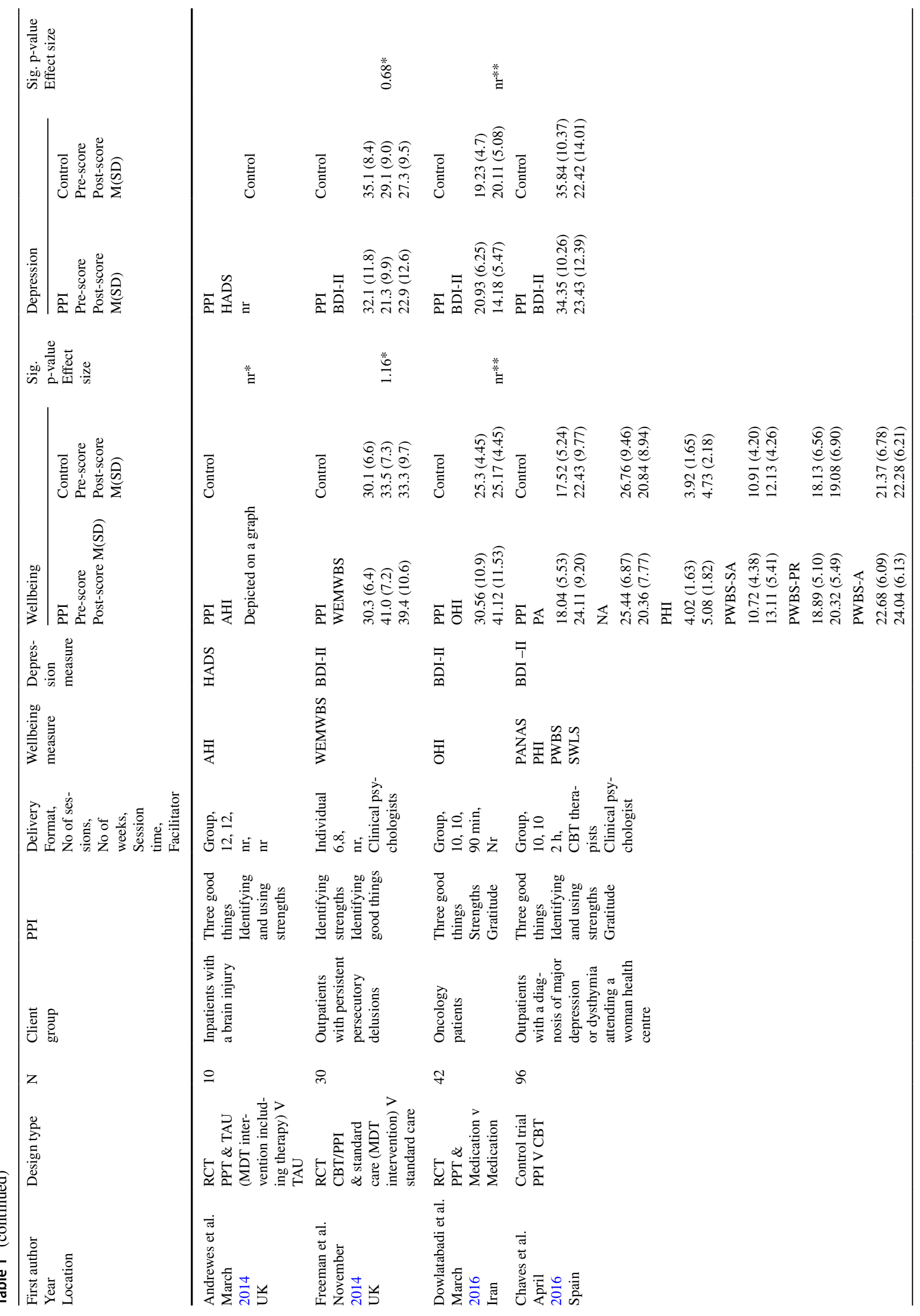




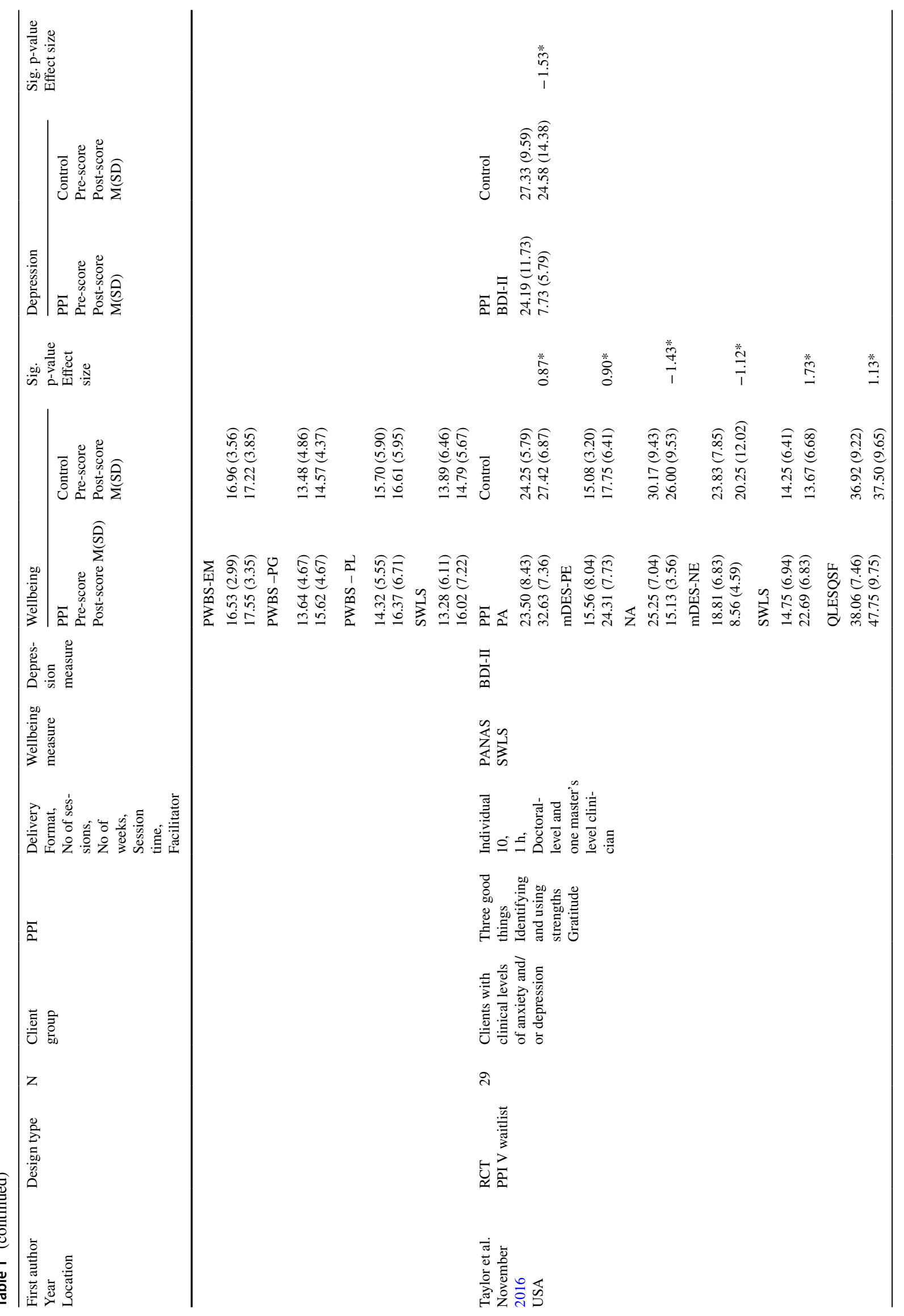




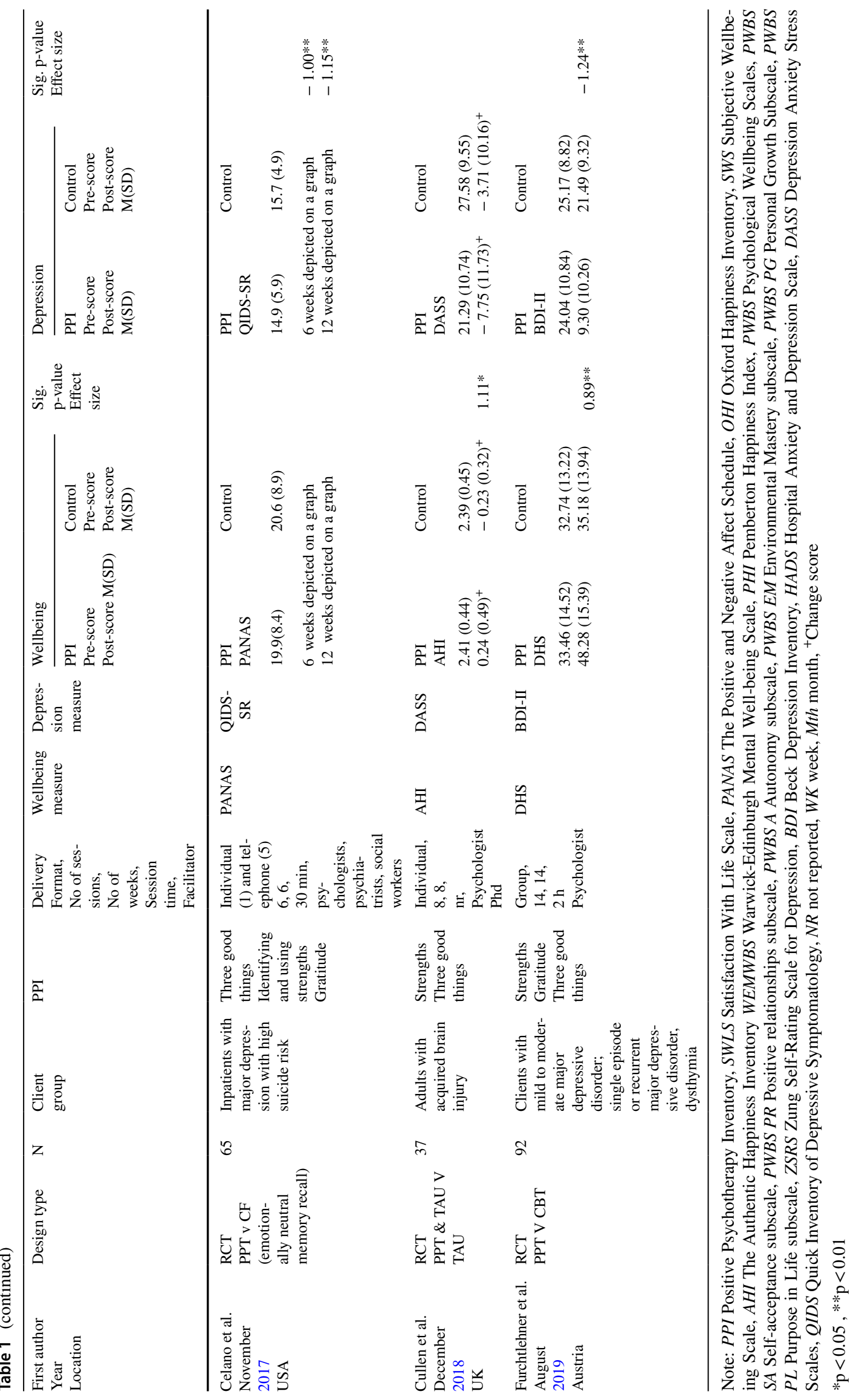


offered a conceptual link between positive psychology theory and wellbeing. Seligman et al. (2006) proffered that happiness consists of positive emotion, engagement and meaning and that PPIs are designed to foster one or more of these elements. Similarly, Dowlatabadi et al. (2016) hypothesised that PPIs enhance positive sentiments, behaviour and cognitions that results in positive effects on happiness. There was greater evidence of a conceptual connection between PPIs and depression, with most studies defining depression in accordance with APA (2013) criteria. For instance, Seligman et al. (2006) advanced that building positive emotion, engagement and meaning alleviates the lack of positive emotion, engagement and meaning inherent in depression. Similarly, Chaves et al. (2016) stipulated that the building of positive emotions and cognitive function counteracts the reduced levels of positive affect in depression. Furchtlehner et al. (2019) too highlighted that there is a lack of positive emotions, engagement, meaning, positive relationships and accomplishment in depression. These hypotheses were therefore derived from the emerging literature base. However, in the majority of cases there was not a clear rationale for applying PPIs. It is noteworthy that two research interventions presented a novel hypothesis that PPIs could improve participants' self-concept.

Despite delivering the Seligman et al. (2005) PPIs, most of the research interventions differed from each other as well as both the original research (Seligman et al., 2005) and the initial clinical intervention (Seligman et al., 2006). Furchtlehner et al. (2019) was the exception in delivering the Seligman et al. (2006) intervention. A minority of research interventions utilized a comprehensive manual whereby the practices were tested by experts in positive psychology alongside the evidence of facilitator training. However, most of the research interventions consisted entirely of PPIs and were not integrated with other therapies. Albeit, the interventions differed in terms of the intensity of the PPIs. For instance: four interventions provided participants with instructions or coaching, five interventions facilitated experiential practice, whist the remaining interventions repeatedly practiced the techniques. It was apparent that a definitive measure of subjective wellbeing was not applied, although established measures of depression were utilized.

In evaluating the RCT design, more than half of the research interventions alluded to randomisation procedures, with the majority evidencing that the groups were similar on salient characteristics prior to intervention. The optimal control of one psychological therapy was utilized in three studies. Crucially, most of the samples were small which negatively impacted on statistical power, although four interventions noted empirical strength with larger samples. The retention rate of the interventions ranged from 71 to $100 \%$, with an overall score of $84 \%$.

\section{Discussion}

\section{Summary of the Main Findings}

The initial evidence indicates that relative to controls, PPIs increase peoples' subjective wellbeing. This finding concurs with previous reviews (Bolier et al., 2013; Sin \& Lyubomirsky, 2009) and contrasts with the inconclusive findings regarding the clinical efficacy of PPIs (Ghosh $\&$ Deb, 2017; Walsh et al., 2017). It also aligns with the primary function of PPIs, which is to increase subjective wellbeing. Fewer studies demonstrated evidence of an impact on depression. However, a closer investigation revealed that the research interventions differed, which prevents the drawing of definitive systematic conclusions. Moreover, a methodological evaluation found recurring issues in: detailing the theoretical underpinning of the studies, delivering the interventions, measuring subjective wellbeing and in implementing the RCT design. Consequently, the methodological quality of the interventions, as measured by the current review, was low. This reiterates previous findings regarding the methodological issues within PPI research (Bolier et al., 2013; Macaskill, 2016).

PPI research is an emerging field of inquiry, reflected by the presence of pilot interventions within this review. It is apparent that clinicians can see the utility of PPIs within multiple contexts. This is to be expected given the transdiagnostic nature of PPIs in fostering peoples' subjective wellbeing. The innovative nature of the work may account for some of the methodological issues and the researchers achieved the preliminary step of offering PPIs within services. Nevertheless, in accordance with the assertions of Gander et al. (2016) the majority of the studies did not provide a theoretical underpinning. A comprehensive definition of wellbeing was also absent and there was a lack of conceptual linking between PPIs and wellbeing. Seligman et al. (2006) conceptualized that PPIs foster positive emotion, engagement and meaning that increases wellbeing, which raises questions regarding whether the processes that lead to subjective wellbeing are the same as the outcomes to be measured or are these distinguishable entities? However, there is since development with the emergence of the PERMA profile measure (Butler \& Kern, 2016) which maps onto the PERMA model in addition to the same form of subjectivewellbeing as delineated by Diener (Goodman et al., 2017). A greater conceptual connection was evident between PPIs and depression. However, overall, there is a gap in the conceptual linking between PPIs, subjective wellbeing, and depression. This reiterates previous findings that PPI mechanisms and the associated outcomes are not set out throughout the studies (Walsh et al., 2017). 
In considering the delivery of PPIs, there is scope to improve the: intervention manuals, training procedures and the supervision arrangements. It is clear that researchers have become more refined in investigating PPIs. However, there were differences in the intensity of and the practicing of the techniques. Ghosh and Deb (2017) too found variation in the application of PPIs. The current research echoes that wellbeing is not clearly defined within the research (Schrank et al., 2013). In terms of the RCT design, the randomization process could be improved, a finding that aligns with other reviews (Bolier et al., 2013, Ghosh \& Deb, 2017). The variability within the controls could also be reduced and the sample sizes could be increased. The high retention rates of PPIs, reiterates previous findings (Lopez-Gomez et al., 2017) regarding the acceptability of PPIs, which could have cost implications.

\section{Strengths and Limitations}

The strengths of this review include that it centred on the Seligman et al. (2005) PPIs as applied in clinical practice to subjective wellbeing and depression. The methodological evaluation was strengthened by drawing on expert guidelines and best practice evidence to create the evaluation grid. Consequently, the quality of the research interventions was examined in depth. The review was limited by the inclusion of different populations, which impacts on intervention comparisons. It also focused on RCT designs, although single case methodologies (Barlow \& Hersen, 1984) can be particularly helpful in understanding individual responses to therapy.

\section{Future Research}

It is suggested for future research to delineate the authentic happiness theory/PERMA model and its evidence-base. A conceptual link between PPIs, wellbeing and depression would also be useful and it is suggested for the research to be guided by the Seligman et al. (2005) hypothesis of increasing subjective-wellbeing. One PPI manual could be piloted and adapted within services. It may also be necessary to adapt measures of subjective wellbeing or a general measure could facilitate recovery. It is recommended for future RCTs: to adhere to the CONSORT guidelines, to recruit larger samples and to use one active control. It would be insightful to undertake another methodological review as the field unfolds. A meta-analysis of the Seligman et al. (2005) PPIs could also be undertaken when the: participant groups, interventions and measures are similar and therefore can be assumed as equivalent.

\section{Conclusion}

To conclude there is emerging evidence that PPIs do significantly differ from controls in increasing peoples' subjective wellbeing and there are fewer studies indicating a difference in reducing depression. However, the research interventions differed, which prevents the drawing of definitive conclusions. A detailed methodological evaluation also revealed issues in: detailing the theoretical underpinning of the studies, delivering the interventions, measuring subjective wellbeing and in implementing the RCT design. The methodological quality of the research interventions, as evaluated by the current review was therefore low. Despite these limitations there is evidence that positive psychology researchers are becoming more refined in investigating PPIs and that people attending different services are responsive to the interventions. However, it is apparent that positive psychology research interventions require greater standardization and improvement in methodological quality in order to rigorously address the question; do positive psychology interventions increase the subjective wellbeing and reduce the clinical depression of people attending services? This is a prerequisite to progressing the health service agendas of offering psychological interventions that: enhance wellbeing, enable recovery and empower people to have a choice of therapies.

Funding None.

\section{Declarations}

Conflict of interest The authors declare that they have no conflict of interest.

Open Access This article is licensed under a Creative Commons Attribution 4.0 International License, which permits use, sharing, adaptation, distribution and reproduction in any medium or format, as long as you give appropriate credit to the original author(s) and the source, provide a link to the Creative Commons licence, and indicate if changes were made. The images or other third party material in this article are included in the article's Creative Commons licence, unless indicated otherwise in a credit line to the material. If material is not included in the article's Creative Commons licence and your intended use is not permitted by statutory regulation or exceeds the permitted use, you will need to obtain permission directly from the copyright holder. To view a copy of this licence, visit http://creativecommons.org/licenses/by/4.0/.

\section{References}

American Psychiatric Association. (2013). Diagnostic and statistical manual of mental disorders (5th ed.). American Psychiatric Publishing. 
Andrewes, H. E., Walker, V., \& O’Neill, B. (2014). Exploring the use of positive psychology interventions in brain injury survivors with challenging behaviour. Brain Injury, 28(7), 965-971.

Asgharipoor, N., Farid, A. A., Arshadi, H., \& Sahebi, A. (2012). A Comparative study on the effectiveness of positive psychotherapy and group cognitive behavioural therapy for the patients suffering from major depressive disorder. Iranian Journal of Psychiatry and Behavioral Sciences, 6(2), 33-41.

Barlow, D., \& Hersen, M. (1984). Single case experimental designs: Strategies for studying behaviour for change. Pergamon Press.

Beck, A. T., Rush, A. J., Shaw, B. F., \& Emery, G. (1979). Cognitive therapy for depression. Guilford Press.

Bolier, L., Haverman, M., Westerhof, G., Riper, H., Smit, F., \& Bohlmeijer, E. (2013). Positive psychology interventions: A meta-analysis of randomized controlled studies. BMC Public Health, 8(13), 119.

Bradburn, N. M. (1969). The structure of psychological wellbeing. National Opinion Research Centre monographs in social research. Aldine.

Butler, J., \& Kern, M. L. (2016). The PERMA-profiler: A brief multidimensional measure of flourishing. International Journal of Wellbeing, 6, 3.

Casellas-Grau, A., Font, A., \& Vives, J. (2014). Positive psychology interventions in breast cancer. A systematic review. PsychoOncology, 23(1), 9-19.

Celano, C. M., Beale, E. E., Mastromauro, C. A., Stewart, J. G., Millstein, R. A., Auerbach, R. P., \& Huffman, J. C. (2017). Psychological interventions to reduce suicidality in high risk patients with major depression: A randomized controlled trial. Psychological Medicine, 47(5), 810-821.

Chakhssi, F., Kraiss, J. T., Sommers-Spijkerman, M., \& Bohlmeijer, E. T. (2018). The effect of positive psychology interventions on well-being and distress in clinical samples with psychiatric or somatic disorders: A systematic review and meta-analysis. BMC Psychiatry, 18(1), 1-17.

Chaves, C., Lopez-Gomez, I., Hervas, G., \& Vazquez, C. (2016). A comparative study on the efficacy of a positive psychology intervention and a cognitive behavioural therapy for clinical depression. Cognitive Therapy and Research, 41(3), 417-433.

Compton, W. C., \& Hoffman, W. (2012). Positive psychology: The science of happiness and flourishing (2nd ed.). Cengage Learning.

Cooke, P. J., Melchert, T. P., \& Connor, K. (2016). Measuring wellbeing: A review of instruments. The Counselling Psychologist, 44(5), 730-757.

Cuijpers, P., Berking, M., Andersson, G., Quigley, L., Kleiboer, A., \& Dobson, K. S. (2013). A meta-analysis of cognitive behavioural therapy for adult depression, alone and in comparison with other treatments. The Canadian Journal of Psychiatry, 58(7), 376-385.

Cullen, B., Pownall, J., Cummins, J., Baylan, S., Broomfield, N., Haig, C., Kersel, D., Murray, H., \& Evans, J. J. (2018). Positive PsychoTherapy in ABI Rehab (PoPsTAR): a pilot randomised controlled trial. Neuropsychological Rehabilitation, 28(1), 17-33.

Diener, E., Emmons, R., Larsen, R., \& Griffin, S. (1985). The satisfaction with life scale. Journal of Personality Assessment, 49(1), 71-75.

Diener, E., Heintzelman, S. J., Kushlev, K., Tay, L., Wirtz, D., Lutes, L. D., \& Oishi, S. (2017). Findings all psychologists should know from the new science on subjective wellbeing. Canadian Psychology, $58(2), 87$.

Dowlatabadi, M. M., Ahmadi, S. M., Sorbi, M. H., Beiki, O., Razavi, T. K., \& Bidaki, R. (2016). The effectiveness of group positive psychotherapy on depression and happiness in breast cancer patients: A randomized controlled trial. Electronic Physician, 8(3), 2175-2180.
Fava, G., Ruini, C., Rafanelli, C., Finos, L., Salmaso, L., Mangelli, L., \& Sirigatti, S. (2005). Wellbeing therapy of generalized anxiety disorder. Psychotherapy and Psychosomatics, 74(1), 26-30.

Field, M., \& Cartwright-Hatton, S. (2015). Essential abnormal and clinical psychology. Sage Publishing.

Fordyce, M. W. (1977). Development of a program to increase personal happiness. Journal of Counselling Psychology, 24(6), 511-521.

Frankl, V. E. (1946). Man's search for meaning. Rider Books.

Freeman, D., Pugh, K., Dunn, G., Evans, N., Sheaves, B., Waite, F., Cernis, E., Lister, R., \& Fowler, D. (2014). An early Phase II randomised controlled trial testing the effect on persecutory delusions of using CBT to reduce negative cognitions about the self: The potential benefits of enhancing self-confidence. Schizophrenia Research, 160(1), 186-192.

Furchtlehner, L. M., Schuster, R., \& Laireiter, A. R. (2019). A comparative study of the efficacy of group positive psychotherapy and group cognitive behavioral therapy in the treatment of depressive disorders: A randomized controlled trial. The Journal of Positive Psychology, 15(6), 832-845.

Gander, F., Proyer, R., Ruch, W., \& Wyss, T. (2013). Strength based positive interventions: Further evidence for their potential in enhancing wellbeing and alleviating depression. Journal of Happiness Studies, 14(4), 1241-1259.

Gander, F., Proyer, R. T., \& Ruch, W. (2016). Positive psychology interventions addressing pleasure, engagement, meaning, positive relationships, and accomplishment increase wellbeing and ameliorate depressive symptoms: A randomized, placebo controlled online study. Frontiers in Psychology, 7, 686.

Gearing, R. E., El-Bassel, N., Ghesquiere, A., Baldwin, S., Gillies, J., \& Ngeow, E. (2011). Major ingredients of fidelity: A review and scientific guide to improving quality of intervention research implementation. Clinical Psychology Review, 31(1), $79-88$.

Ghosh, A., \& Deb, A. (2017). Positive psychology interventions for chronic physical illnesses: A systematic review. Psychological Studies, 62(3), 213-232.

Goodman, F. R., Disabato, D. J., Kashdan, T. B., \& Kauffman, S. B. (2017). Measuring wellbeing: A comparison of subjective wellbeing and PERMA. The Journal of Positive Psychology, 13, 321-332.

Green, L. S., Oades, L. G., \& Grant, A. M. (2006). Cognitive-behavioral, solution-focused life coaching: Enhancing goal striving, well-being, and hope. The Journal of Positive Psychology, 1(3), 142-149.

Guy, A., Loewenthal, D., Thomas, R., \& Stephenson, S. (2012). Scrutinising NICE: the impact of the national institute for health and clinical excellence guidelines on the provision of counselling and psychotherapy in primary care in the UK. Psychodynamic Practice, 18(1), 25-50.

Hone, L. C., Jarden, A., \& Schofield, G. M. (2015). An evaluation of positive psychology intervention effectiveness trials using the re-aim framework: A practice-friendly review. The Journal of Positive Psychology, 10(4), 303-322.

Iddon, J. E., Dickson, J. M., \& Unwin, J. (2016). Positive psychological interventions and chronic non cancer pain: A systematic review of the literature. International Journal of Applied Positive Psychology, 1(1-3), 133-157.

Jadad, A. R., Alejandro, R., Moore, R. A., Carroll, D., Jenkinson, C., Reynolds, D. J., Gavaghan, D. J., \& McQuay, H. J. (1996). Assessing the quality of reports of randomized clinical trials: Is blinding necessary? Controlled Clinical Trials, 17(1), 1-12.

Johnson, J., \& Wood, A. M. (2017). Integrating positive and clinical psychology: Viewing human functioning as continua from positive to negative can benefit clinical assessment, interventions 
and understandings of resilience. Cognitive Therapy and Research, 41(3), 335-349.

Kazdin, A. E. (1995). Preparing and evaluating research reports. Psychological Assessment, 7(3), 228-237.

Liebherz, S., Machmutow, K., Jansen, A., Meister, R., Watzke, B., Härter, M. C., \& Kriston, L. (2017). Continuation and maintenance treatments for persistent depressive disorder. The Cochrane Database of Systematic Reviews. https://doi.org/10. 1002/14651858.CD012855

Linley, A., Joseph, S., \& Boniwell, L. (2003). Positive psychology special issue. The Psychologist, 16(3), 126-143.

Lopez-Gomez, I., Chaves, C., Hervas, G., \& Vazquez, C. (2017). Comparing the acceptability of a positive psychology intervention versus a cognitive behavioural therapy for clinical depression. Clinical Psychology \& Psychotherapy, 24(5), 1029-1039.

Macaskill, A. (2016). Review of positive psychology applications in clinical medical populations. Healthcare, 4(3), 66.

Magyar-Moe, J., Owens, R., Scheel, M., Buki, L., \& Sheldon, K. (2015). Special issue: Applications of positive psychology. Counselling Psychologist, 43(4 \& 5), 493-788.

Maslow, A. (1943). A theory of human motivation. Psychological Review, 50, 370-396.

Medical Research Council. (2000). A framework for the development and evaluation of RCTs for complex interventions to improve health. MRC.

Moher, D., Liberati, A., Tetzlaff, J., Altman, D. G., \& The, P. G. (2009). Preferred reporting items for systematic reviews and meta-analyses: the PRISMA Statement. PLoS Medicine, 6, e1000097.

Mongrain, M., \& Anselmo-Matthews, T. (2012). Do positive psychology exercises work? A replication of Seligman et al. 2005. Journal of Clinical Psychology, 68(4), 382-389.

Nathan, P., \& Gorman, J. M. (2007). Challenges to implementing evidence-based treatments. In P. E. Nathan \& J. M. Gorman (Eds.), A guide to treatments that work (4th ed., pp. 1-22). Oxford University Press.

Olive, S. A., Macedon, L. G., Galotti, I. C., Fuentes, J., Stanton, T., \& Magee, D. J. (2008). Scales to assess the quality of randomized controlled trials: A systematic review. Physical Therapy, 88(2), $156-175$.

Payton, A. (2009). Mental health, mental illness, and psychological distress: Same continuum or distinct phenomena? Journal of Health and Social Behaviour, 50(2), 213-227.

Peterson, C. (2005). Authentic happiness inventory. Pennsylvania USA: University of Pennsylvania. Retrieved January 6, 2018, from https://www.authentichappiness.sas.upenn.edu.

Peterson, C. (2006). A primer in positive psychology. Oxford University Press.

Peterson, C., \& Seligman, M. E. (2004). Character strengths and virtues: A handbook and classification. Oxford University Press.

Pietrowsky, R., \& Mikutta, J. (2012). Effects of positive psychology interventions in depressive patients - a randomized control study. Psychology, 3(12), 1067-1073.

Querstret, D., \& Cropley, M. (2013). Assessing treatments used to reduce rumination and/or worry: A systematic review. Clinical Psychology Review, 33(8), 996-1009.

Radloff, L. S. (1977). The CES-D scale: Self-report depression scale for research in the general population. Applied Psychological Measurement, 1(3), 385-401.

Rashid, T. (2015). Positive psychotherapy: A strength based approach. The Journal of Positive Psychology, 10(1), 25-40.

Rogers, C. R. (1961). On becoming a person: A therapist's view of psychotherapy. Houghton Mifflin Company.

Rychetnik, L., Frommer, M., Hawe, P., \& Shiell, A. (2002). Criteria for evaluating evidence on public health interventions. Journal of Epidemiology \& Community Health, 56(2), 119-127.
Santos, V., Paes, F., Pereira, V., Carrion, O. A., Silva, A. C., Carta, M. G., Nardi, A. E., \& Machado, S. (2013). The role of positive emotion and contributions of positive psychology in depression treatment: Systematic review. Clinical Practice and Epidemiology in Mental Health, 9, 221-237.

Schrank, B., Bird, V., Tylee, A., Coggins, T., Rashid, T., \& Slade, M. (2013). Conceptualising and measuring the wellbeing of people with psychosis: Systematic review and narrative synthesis. Social Science and Medicine, 92, 9-21.

Schulz, K. F., Altman, D. G., \& Moher, D. (2010). CONSORT 2010 statement: Updated guidelines for reporting parallel group randomised trials. BMC Medicine, 8(1), 18.

Seligman, M. (1972). Learned helplessness. Annual Review of Medicine, 23, 407-412.

Seligman, M. E. (2002). Authentic happiness. Simon \& Schuster.

Seligman, M. E. (2012). Flourish: A visionary new understanding of happiness and wellbeing. Nicholas Brealey.

Seligman, M., \& Csikszentmihalyi, M. (2000). Positive psychology an introduction. American Psychologist, 55(1), 5-14.

Seligman, M. E., \& Rashid, T. (2014). Positive psychotherapy. In D. Wedding \& R. J. Corsini (Eds.), Current psychotherapies (pp. 461-449). Cengage Learning.

Seligman, M. E., Steen, T., Park, N., \& Peterson, C. (2005). Positive psychology progress: Empirical validation of interventions. American Psychologist, 60(5), 410-421.

Seligman, M. E., Rashid, T., \& Parks, A. (2006). Positive psychotherapy. American Psychologist, 61(8), 774-778.

Sin, N., \& Lyubomirsky, S. (2009). Enhancing well-being and alleviating depressive symptoms with positive psychology interventions: A practice-friendly meta-analysis. Journal of Clinical Psychology, 65(5), 467-487.

Taylor, C. T., Lyubomirsky, S., \& Stein, M. B. (2016). Upregulating the positive affect system in anxiety and depression: Outcomes of a positive activity intervention. Depression and Anxiety, 34(3), 267-280.

Tugade, M. M., \& Fredrickson, B. L. (2004). Resilient individuals use positive emotions to bounce back from negative emotional experiences. Journal of Personality and Social Psychology, 86(2), 320-333.

Vazquez, C., Rahona, J. J., Gomez, D., Caballero, F. F., \& Hervas, G. (2015). A national representative study of the relative impact of physical and psychological problems on life satisfaction. Journal of Happiness Studies, 16(1), 135-148.

Walsh, S., Cassidy, M., \& Priebe, S. (2017). The application of positive psychotherapy in mental health care: A systematic review. Journal of Clinical Psychology, 73(6), 638-651.

Watson, D., \& O'Hara, M. W. (2017). Understanding the emotional disorders: A symptom level approach based on the IDAS II. Oxford University Press.

Watson, D., Clark, L. A., \& Tellegen, A. (1988). Development and validation of brief measures of positive and negative affect: The PANAS Scales. Journal of Personality and Social Psychology, 54(6), 1063-1070.

Wood, A., \& Tarrier, N. (2010). Positive clinical psychology. Clinical Psychology Review, 30(7), 819-906.

Wood, A. M., Taylor, P. J., \& Joseph, S. (2010). Does the CES-D measure a continuum from depression to happiness? Comparing substantive and artifactual models. Psychiatry Research, 177(1), $120-123$.

Publisher's Note Springer Nature remains neutral with regard to jurisdictional claims in published maps and institutional affiliations. 